\title{
How to Extract the Newtonian Viscosity from Capillary Breakup Measurements In a Filament Rheometer
}

\author{
Gareth H. McKinley, Anubhav Tripathi \\ Dept. of Mechanical Engineering, M.I.T. \\ Cambridge, MA 02139, U.S.A.
}

February 14, 2000

\begin{abstract}
The liquid filament microrheometer originally described by Bazilevsky et al. (1990) provides a simple way of extracting material parameters for Newtonian and viscoelastic fluids from measurements of the capillary breakup of a thin fluid thread. However, there is an unresolved discrepancy in the value of the Newtonian viscosity obtained from the experimental data when using the existing theoretical analysis. We demonstrate how to correctly analyze measurements of the midpoint radius and present a simple formula that enables one to obtain quantitative values for the Newtonian viscosity for a range of viscous fluids. The validity of this correction is supported by numerical simulations and experiments with a number of viscous Newtonian fluids. In addition we analyze the role of gravitational body forces on modifying the dynamics of capillary thinning of a Newtonian liquid filament. Finally, we show how such capillary breakup devices may be used to make quantitative time-resolved measurements of changes in the viscosity of hygroscopic materials or fluids with a volatile solvent component that are exposed to an ambient atmosphere.
\end{abstract}




\section{Introduction}

Filament rheometers are becoming an increasingly common means of extracting information about the extensional rheological properties of fluids. Data is extracted from these devices through quantitative observations of the evolution of a thin fluid filament under the combined action of viscous, elastic and capillary forces. In the original 'falling plate' configuration of Matta \& Tytus (1990) the sample is subject to a constant load that is imparted by a known weight attached to one of the endplates constraining the sample. In a filament stretching device (Tirtaatmadja \& Sridhar 1993; Spiegelberg et al. 1996) a known (exponential) displacement profile is imposed and quantitative measurements of the tensile force along the fluid column, $F_{z}(t)$, and midpoint filament radius, $R_{\text {mid }}(t)$, are followed in time. In the liquid filament rheometer described by Bazilevsky et al. (1990), a 'necked' liquid bridge configuration is generated by rapidly separating two cylindrical plates a small distance, and the evolution of the midpoint radius, $R_{\text {mid }}(t)$, is subsequently followed in time.

In this latter class of device it is not necessary to impose a continued exponential elongation and the force is not measured; instead the system selects its own dynamics so that the viscous, elastic, gravitational and capillary forces balance each other. Large strains are attained for the Lagrangian fluid elements near the initial neck as the filament approaches the singular event of breakup which results in a topological transition into two disconnected fluid domains. As a consequence of this difference, filament rheometers which focus on the capillary breakup regime are much simpler and cheaper to build than filament stretching devices; however, analysis of the resulting data is more complex since a known homogeneous deformation profile is not imposed and it is necessary to understand exactly what deformation the system selects (and also why it selects this response) if one desires to recover accurate values for material parameters such as the fluid viscosity or characteristic relaxation time.

In early work, Schümmer \& Tebel $(1980,1983)$ described an elongational rheometer in which one manually analyzed photographic images of the capillary instability of periodicallyforced free jets of polymeric fluids. From these observations they were able to extract values of a deformation-rate-dependent fluid relaxation time and an apparent elongational viscosity. Their photographs of the jet evolution at long times showed the development of the well-known 'beads-on-a-string' morphology (Goldin et al. 1969) which was subsequently described in numerical and analytical work by Entov \& Yarin (1984) and by Bousfield et al. (1986). Further details of early experimental and theoretical work are discussed in the review by Bogy (1979) and in the comprehensive monograph by Yarin (1993). 
Bazilevsky et al. (1990) were the first to describe a simple rheometric device using a 'real-time' optical method for monitoring the evolving diameter of a fluid column undergoing capillary instability. They also presented a framework for analyzing the resulting single point measurement of the midpoint diameter that is appropriate for both Newtonian and viscoelastic fluids described by an Oldroyd-B constitutive equation. This analysis was extended to consider the effect of multiple relaxation modes and finite extensibility of polymer chains by Entov \& Hinch (1997). In this analysis, experimental observations of the slenderness of the structures that develop during the breakup dynamics motivated the neglect of axial curvature along the necked fluid column, and the liquid bridge is approximated as an axially uniform thread that can be characterized simply by its midpoint radius $R_{\text {mid }}(t)$. For a Newtonian fluid of viscosity $\eta_{s}$ and surface tension $\sigma$, a local force balance, and elimination of the fluid pressure, leads to the following evolution equation for the midpoint radius,

$$
3 \eta_{s}\left(-\frac{2}{R_{\text {mid }}} \frac{d R_{\text {mid }}(t)}{d t}\right)=\frac{\sigma}{R_{\text {mid }}(t)} .
$$

In developing this evolution equation, the total longitudinal stress along the fluid thread is assumed to be zero at all times since the column is connected to large quasi-static fluid reservoirs which are themselves attached to the rigid endplates of the device. The term in parentheses can be identified as the extensional deformation rate of the Lagrangian fluid element at the midplane of the column where the radius is measured. Equation (1) can also be obtained from the dispersion relation for the growth of infinitesimal disturbances on the surface of a cylindrical viscous jet in a vacuum. Lord Rayleigh's linear stability analysis of the Stokes' equations showed that, in the inertialess limit, the fastest growing mode corresponds to an infinite wavelength disturbance with (dimensional) growth rate $\omega_{\max }=\sigma /\left(6 \eta_{s} R_{j e t}\right)$ where $R_{j e t}$ is the radius of the cylindrical jet (see the reviews of Bogy, 1979 and Eggers 1997 for further details).

Measurements of the rate of decrease in the measured filament radius thus predict a constant slope $-\left(\sigma / 6 \eta_{s}\right)$, and integration of eq. (1) leads immediately to a linearly decreasing profile which can be written in several useful forms:

$$
R_{\text {mid }}(t)=R_{1}-\frac{\sigma}{6 \eta_{s}} t=\frac{\sigma}{6 \eta_{s}}\left(t_{c}-t\right)
$$

where $R_{1}$ is the initial radius of the thread at time $t=0$, and $t_{c}$ is the critical time of the breakup event, which is determined, in this analysis, directly by the initial conditions to be $t_{c}=6 \eta_{s} R_{1} / \sigma$. In obtaining eq. (2), we have effectively assumed that the fastest growing mode determined from linear stability analysis of a cylindrical fluid jet remains dominant all the way to the final breakup event. 
Liang \& Mackley used the microfilament rheometer to study the breakup dynamics of a number of concentrated viscoelastic solutions of polyisobutylene in Decalin, plus a viscous Newtonian PDMS oil. For the Newtonian fluid, a linear decrease in radius with time was observed and they used the analysis above to obtain values of the ratio $\left(\sigma / \eta_{s}\right)$ which may be termed a capillary velocity and is a measure of the characteristic rate of necking in a Newtonian fluid thread. However, when they used this ratio with a separate rheometric measurement of the solvent viscosity they obtained a surface tension value of $\sigma^{(P D M S)} \approx 9.74 \times 10^{-3} \mathrm{~N} / \mathrm{m}$ which is approximately a factor of two below accepted literature values. Recently, Kolte \& Szabo (1999) presented a new variant of a capillary breakup rheometer and attempted to recover values of the steady shear viscosity for a Newtonian polybutene oil. They also observed a linear decrease in the measured midpoint radius; however when they used eq. (2) to extract the solvent viscosity they found a very marked discrepancy with the value independently measured in a torsional rheometer. A full time-dependent numerical simulation of the evolution of the fluid column undergoing capillary breakup showed excellent agreement with the experimental observations, and furthermore showed that the effects of a gravitational body force acting axially along the necked fluid column could not safely be neglected. The authors thus concluded that it is not possible to use the simple analysis above to extract the Newtonian fluid viscosity.

Interestingly, the difficulty in obtaining accurate material parameters for highly elastic fluids is less pronounced. Theoretical work (Bousfield et al. (1986), Bazilevsky et al. (1990); Renardy (1994)) and the subsequent analysis of Entov \& Hinch for FENE-P dumbbell constitutive models shows that following a rapid initial viscous-dominated phase, there is an intermediate time-scale in which the dynamics of the filament drainage are governed by a balance between surface tension and elasticity, rather than fluid viscosity. In this regime, the filament radius decreases exponentially as $R_{\text {mid }}(t)=R_{1} \exp \left(-t / 3 \lambda_{c}\right)$ where $\lambda_{c}$ is a characteristic relaxation time governing the capillary breakup. The question remains how to relate $\lambda_{c}$ to the linear viscoelastic spectrum of the fluid determined, for example, from small amplitude linear viscoelasticity? Kolte \& Szabo (1999) show for a semi-dilute PIB/PB Boger fluid that in fact $\lambda_{c}$ is closely related to the longest relaxation time, $\lambda_{1}$ of the fluid. They also elegantly show how the effects of a radial inhomogeneity in the stretch can account for the remaining discrepancy between $\lambda_{c}$ and $\lambda_{1}$. Recent numerical simulations for filament stretching rheometers show that this radial inhomogeneity is a strong function of the initial shape of the fluid sample (Harlen, 1996) and also the initial aspect ratio (Yao et al. 1999). We have recently demonstrated that by choosing an appropriate aspect ratio it is, in fact, possible to use measurements of the exponential decrease in radius with time to quantitatively determine the longest (Rouse/Zimm) relaxation time for ideal elastic fluids (consisting of dilute solutions of monodisperse 
polystyrene) and the approximate scaling of the steady-state elongational viscosity with molecular weight (Anna \& McKinley, 1999).

The inability to obtain the constant viscosity for a Newtonian or weakly elastic fluid is obviously a drawback inhibiting the use of such an instrument in rheometry, since it is undesirable to have to resort to a full numerical simulation every time one wishes to extract a viscometric property from experimental observations. Similar difficulties are faced in determining the interfacial tension from observations of droplet breakup, and Tjahjadi et al. (1994) performed an extensive series of computations to produce a set of numerical tables that can be used in analyzing experimental observations. The purpose of this short communication is to demonstrate that in the microfilament rheometer it is possible to use a simple analytic expression to extract quantitative values of the Newtonian viscosity, at least for viscous fluids ( $\eta_{s} \geq 0.1$ Pa.s). In $\S 2$ we outline the theoretical framework we use for computations and briefly summarize existing theoretical results for capillary breakup of slender fluid jets. In $\S 3$ we present numerical calculations, both with and without an axial body force, that show how the late stages of the filament drainage are simply, but substantially modified, from eq. (2). Using this new approach we determine the viscosity from experimental observations with three different Newtonian fluids and show that quantitative agreement can be obtained with values determined directly from steady shear rheometry. 


\section{Theoretical Background}

In order to demonstrate why the discrepancy between experimentally-measured and theoretically-expected values of $\sigma / \eta_{s}$ for a Newtonian fluid arises, we first summarize a convenient theoretical framework for presenting our numerical and experimental results.

\subsection{Problem Formulation}

The basic configuration of a filament rheometer is shown in Figure 1. A small volume of fluid is initially extruded from a syringe through a flexible capillary tube. The sample is constrained axially between two smooth coaxial disks of radius $R_{0}$ and forms a liquid bridge configuration that is nominally cylindrical in shape. The precise shape is determined by satisfying the Young-Laplace equation and is a function of the aspect ratio $\Lambda_{0}=L_{0} / R_{0}$, the volume of fluid contained between the plates and the gravitational body force and surface tension (Slobozhanin et al. 1993; Szabo, 1997). The relative magnitudes of the latter quantities can be expressed in terms of the Bond number $B o=\rho g R_{0}^{2} / \sigma$ where $\rho$ is the fluid density and $\sigma$ is the surface tension. For $B o=0$, the maximum stable length of a cylindrical liquid bridge of volume $V_{0}=\pi R_{0}^{2} L_{0}$ is $L_{\max }=2 \pi R_{0}$ as first noted by Plateau (1863). The maximum stable length decreases rapidly from this Plateau stability limit as the Bond number increases or the volume of fluid contained between the plates decreases (Gillette \& Dyson 1970; Slobozhanin, 1993).

At time $t=0$, one or both of the plates is set in motion with velocity $\dot{L}_{p}(t)$ and the liquid bridge is deformed into a necked but axisymmetric configuration (cf. Fig. 1(b)). The radius $R_{\text {mid }}(t)$ at the axial midplane is either measured directly with a laser micrometer or obtained from digitized images of the entire column profile. If inertial effects and viscous stresses in the external fluid are negligible then this necked configuration is symmetric about the midplane; however, if these effects become significant, then more complex shapes arise (Berg et al. 1994; Gaudet et al. 1996). In a filament stretching device the imposed displacement is exponential in nature and the force exerted on one of the plates is monitored as a function of time in order to extract the extensional viscosity (Szabo, 1997). However in a device such as the Microfilament Rheometer described by Bazilevsky et al. the endplate motion is not controlled precisely but rather serves to provide a step displacement of the end plate to a new position $L_{1}$ which exceeds the Plateau stability criterion, $L_{\max }$ at a time $t_{1}=t_{0}+\delta t$. In this respect the device functions as the extensional equivalent of the step-strain test in conventional rheometry. Once this new unstable necked configuration has been established (see Fig. 1(c)), the midpoint radius is monitored as a function of time using the laser micrometer.

The Plateau stability limit is determined from a quasi-static energy balance and for a Newtonian fluid thus depends only on one material property; the surface tension. By contrast, the dynamics of the drainage of the thin fluid column and the ultimate rupture of the liquid bridge 
into two or more droplets are governed by the viscous and elastic properties of the fluid. A complete understanding of this nonlinear process has only been developed over the past decade, and recent developments are elegantly summarized in the comprehensive review of Eggers (1997). In this work, Eggers derives the governing equations of motion for a slender fluid filament (i.e. a filament in which axial curvature terms are smaller than the azimuthal curvature) and shows how, in the limit of vanishing inertial effects, they can be integrated once to give a set of equations describing the forces acting on one-dimensional Lagrangian fluid 'slices'. A Lagrangian formulation of the governing equations for fiber-spinning in the absence of surface tension and subject to a constant axial force is discussed by Yarin (1993); however, the appropriate system of equations for capillary-driven breakup of a fluid thread with a timevarying tensile force was first discussed and analyzed by Renardy (1995). We have recently shown how these one-dimensional equations can accurately reproduce full two-dimensional, time-dependent simulations with both the Newtonian and Giesekus constitutive equations (Yao et al. 1998). As originally formulated, the equation derived by Renardy is written in terms of a Lagrangian stretch for each axial slice of the column defined as

$$
\left.S\left(Z_{0}, t\right) \equiv \partial z / \partial Z_{0}=R_{0}^{2} / R\left(z\left(Z_{0}\right), t\right)\right)^{2},
$$

where $z\left(Z_{0}, t\right)$ denotes the Lagrangian position at time $t$ of the one-dimensional fluid 'slice' located originally at axial position $Z_{0}$ at time $t_{0}$. In experimental measurements of the midpoint radius or in digitized video images of liquid bridge shapes as a function of time, the radial profile $R\left(z\left(Z_{0}, t\right), t\right)$ is the primitive variable. If the stretch $S$ is eliminated from the slender filament equation using equation (3) and the equation is extended to incorporate the effects of higher order axial curvature terms (Eggers, 1997) and also an axial gravity field $\boldsymbol{g}=-g \boldsymbol{\delta}_{z}$ we obtain the following stress balance

$$
3 \eta_{s}\left\{-\frac{2}{R}\left(\frac{\partial R}{\partial t}\right)_{Z_{0}}\right\}=\frac{F_{z}(t)}{\pi R^{2}}-\left[T_{z z}-T_{r r}\right]-\frac{\sigma}{R}\left\{\frac{1}{\left[1+\left(R^{\prime}\right)^{2}\right]^{1 / 2}}+\frac{R R^{\prime \prime}}{\left[1+\left(R^{\prime}\right)^{2}\right]^{3 / 2}}\right\}+\frac{\rho g R_{0}^{2} Z_{0}}{R^{2}}
$$

where the term in braces on the left indicates the strain rate in each fluid slice, and $F_{\mathrm{z}}$ is the (unknown) tensile force acting along the column. In the absence of fluid inertia, this latter term is constant along the column axis but can vary in time. The term in square brackets on the right indicates the contribution of the polymeric stresses from an appropriate viscoelastic constitutive equation. This term is identically zero for a Newtonian fluid. The term in braces on the right is the mean curvature of the filament and a prime indicates a spatial derivative with respect to the current axial position $z$. In the original formulation of Renardy the higher order derivatives arising from axial curvature were omitted; however, Eggers (1997) discusses how incorporation of these terms leads to more stable numerical methods. For the filament rheometer 
used in the present work these higher order terms are small over almost the entire axial length of the liquid bridge, except near the fixed endplates. In this region, axial curvature becomes significant (Shipman et al. 1991; Spiegelberg et al. 1996; Kolte \& Szabo 1999) and should thus be retained; however in these regions other effects that cannot be captured by the onedimensional theory also become important, most notably the two-dimensional shearing flow arising from the no-slip boundary condition on the endplates. For clarity we therefore omit these terms from the following equations, although it is straightforward to retain the higher order axial curvature terms in the one-dimensional numerical simulations and examine their influence.

If we scale all lengths in eq. (4) with the fixed radius of the endplates, $R_{0}$, and express the radius in terms of the Lagrangian stretch $S$, non-dimensionalize time with the capillary scale $t_{\text {cap }}=\eta_{S} R_{0} / \sigma$ and scale stresses with $\sigma / R_{0}$, then the evolution of the viscous Newtonian liquid bridge can be written in the compact form

$$
\frac{3}{S^{2}}\left(\frac{\partial S}{\partial t}\right)_{\zeta_{0}}=f(t)-\frac{1}{\sqrt{S}}\{1+\ldots\}+B o \zeta_{0},
$$

where $\zeta_{0}=Z_{0} / R_{0}$ indicates the dimensionless Lagrangian label and $f(t)=F_{z}(t) /\left(\pi \sigma R_{0}\right)$ is the dimensionless axial force. To close the equation set, $f$ must be determined using an integral constraint along the filament. Using eq. (3) to relate the imposed axial rate of deformation, $\dot{L}_{p}(t)$, to the change in the total stretch of the fluid column this becomes

$$
f(t) \equiv \frac{F_{z}(t)}{\pi \sigma R_{0}}=\frac{3 \dot{L}_{p}(t) / R_{0}+\int_{0}^{\Lambda_{0}} S^{3 / 2}\{1+\ldots\}-S^{2} B o \zeta_{0} d \zeta_{0}}{\int_{0}^{\Lambda_{0}} S^{2} d Z_{0}}
$$

We use equations (5) and (6) in the numerical simulations presented in $\S 3.1$ below.

\subsection{Reconciling Different Expressions for The Rate of Necking}

Comparing equations (4) and (1) it is clear that the slender filament equation of Renardy (describing the situation shown in Figure 1(c)) is different to the equation derived by Entov \& Hinch for an axially uniform liquid thread (as depicted in Figure 1(d)) even in the limit $B o=0$. This discrepancy was also noted in the recent work of Kolte \& Szabo (1999) and arises from the assumption of zero longitudinal fluid stress in the local force balance described by eq. (1). The apparent inconsistency between the two formulations can be resolved if two criteria are satisfied; (i) axial gradients along the liquid bridge become increasingly negligible at later times so that $R(z, t) \rightarrow R_{\text {mid }}(t)$, and (ii) if the net tensile force in the thread approaches a specific value as the radius decreases which is given by 


$$
\lim _{R_{\text {mid }} \rightarrow 0} F_{z}(t) \rightarrow 2 \pi \sigma R_{\text {mid }}(t) .
$$

This special limit can readily be checked using the Lagrangian numerical formulation described above by taking the ratio of the computed axial force to the midpoint radius at each time step. We thus define a new dimensionless variable $X(t)$ by

$$
X(t) \equiv \frac{F_{z}(t)}{2 \pi \sigma R_{\text {mid }}(t)}=\frac{1}{2} f \sqrt{S_{\text {mid }}},
$$

where $S_{\text {mid }}(t)=R_{0}{ }^{2} / R_{\text {mid }}{ }^{2}$ is the dimensionless mid-point stretch for the Lagrangian label at $\zeta_{0}=0.5 \Lambda_{0}$.

In the numerical calculations we thus set $B o=0$ and examine how the product of the (vanishing) force $f$ and (diverging) mid-point stretch $S_{\text {mid }}$ varies in time. If the value of $X$ determined from these calculations is a constant, then substitution of $R(z, t) \rightarrow R_{\text {mid }}(t)$ plus eq. (8) into eq. (4) followed by integration in time leads to the following prediction for the time evolution in the midpoint radius:

$$
R_{\text {mid }}(t)=R_{1}-\frac{(2 X-1)}{6} \frac{\sigma}{\eta_{s}} t
$$

It is clear from eq. (9) that the solution arising from a local force balance for a Newtonian fluid (eq. 2) corresponds to $X=1$. The nonlocal effects arising from axial variations in the shape of the filament are encoded in the time-evolving tensile force, $F_{z}(t)$ (and hence from eq. (8) in $X(t)$ ) and can be determined using the integral constraint given by eq. (6).

In the past decade a number of self-similar solutions to the equations of motion for a slender fluid filament have been discovered, each of which is valid for a different dynamical regime. Details of these similarity solutions are discussed in depth elsewhere (Eggers 1997) but, remarkably, when the appropriate similarity variables are re-expressed in terms of dimensional variables and the numerical solutions are evaluated specifically for the minimum radius, one always obtains an equation of the form given by (9). The only change in the prediction of each similarity solution is the time-rate of change in the midpoint radius. Values of the constant $X$ and the slope $(2 X-1) / 6$ for these different similarity solutions are summarized in Table 1.

In 1995, Renardy gave parameter ranges for three different classes of similarity solution. The dimensionless stretch $S$ in each class of solution diverged with a different critical exponent, but no numerical values of $X$ were reported in this work. Of the three solutions presented by Renardy only the final one is consistent with a smooth initial filament profile. Papageorgiou (1995) subsequently determined numerically the value $X=0.7127$ for the case of an inertialess viscous filament with a smoothly necked profile. In this similarity solution to the unsteady Stokes equations, the similarity exponent characterizing the rate of contraction in the axial scale of the solution is much smaller (by a factor of 0.175 ) than the one for the radial scale and thus 
the filament rapidly becomes increasingly slender. However it never becomes truly axially uniform as required by eqs. (1) \& (2). Eggers $(1993 ; 1997)$ shows that in the very final stages of break up inertial effects can no longer be neglected and the time-rate of change in the filament profile crosses over to a universal similarity solution that balances inertial, capillary and viscous effects. Very recently, Brenner et al. (1996) have shown that, in fact, there are countably infinite similarity solutions to the slender filament equations with values of $X$ approximately determined by the formula $X \approx 1 /(60 n-27)$ for $n=1,2 \ldots$ Numerical calculations showed that the solution of Eggers is special (and would be the one typically observed in experiments with fluids in which inertial effects can not be neglected) since it is the least unstable to finite amplitude perturbations.

\begin{tabular}{lcccl}
\hline & & $X$ & $(2 X-1) / 6$ & Notes \\
\hline \hline Local Force Balance & $R e=0$ & 1 & $1 / 6$ & Axially uniform thread \\
Renardy (1995) & $R e=0$ & - & - & Three different similarity regimes \\
Papageorgiou (1995) & $R e=0$ & 0.7127 & 0.0709 & Valid for viscous fluid filaments \\
Eggers (1993; 1997) & $R e>0$ & 0.5912 & 0.0304 & Universal similarity solution \\
Brenner et al. (1996) & $R e>0$ & 0.5912 & 0.0304 & Countably infinite similarity \\
& & 0.5324 & 0.0108 & solutions \\
& & $\vdots$ & $\vdots$ & \\
\hline
\end{tabular}

Table 1 Summary of the different similarity solutions obtained for the breakup of slender columns of a viscous Newtonian fluid. Here the appropriate Reynolds number quantifying the importance of inertia in the necking filament is in fact the inverse of the square of the Ohnesorge number, $R e=O h^{-2}=\left(\rho \sigma R_{0} / \eta_{s}{ }^{2}\right)$ (see Eggers, 1997 for further discussion).

From a pragmatic viewpoint, the importance of the results in Table 1 to the microfilament rheometer is the following: if an experiment is performed and the midpoint radius $R_{\text {mid }}(t)$ is indeed found to decrease linearly in time, then the value of the ratio $\sigma / \eta_{s}$ determined from regression of the data will depend critically on what value of $X$ it is appropriate to use in the 
analysis. To date the experimental results analyzed by Bazilevsky et al. (1990), Liang \& Mackley (1994) and by Kolte \& Szabo (1997) have all assumed implicitly that $X=1$ in accordance with the local force balance solution of eqs. (1)-(2). We now proceed to show that in fact, for typical experimental conditions, the most appropriate value is actually $X=0.7127$ in accordance with the self-similar solution of Papageorgiou.

\section{Results}

\subsection{Numerical Simulation of Breakup in a Microfilament Rheometer}

We first investigate numerically the evolution of a viscous Newtonian liquid bridge using equations (5) \& (6). In particular, we are interested in how the solution for $R_{\text {mid }}$ evolves in time and the corresponding numerical value of the ratio $X(t)$ defined in eq. (8). To begin with we ignore gravitational effects and set $B o=0$. For the purposes of comparison with experiment we choose Newtonian fluid parameters of $\eta_{s}=11.5 \mathrm{~Pa}$.s and surface tension $\sigma=29 \times 10^{-3} \mathrm{~N} / \mathrm{m}$ to match the polybutene oil studied in the experiments and numerical simulations of Kolte \& Szabo. As an initial necked configuration at time $t_{1}$ (immediately after the plates have stopped separating), we choose the lubrication solution described by Spiegelberg et al. (1996) with $N=1001$ Lagrangian points distributed axially along the initially cylindrical free surface. Hence, for the geometric conditions given in Kolte \& Szabo (1997), the midpoint radius at the beginning of the simulation is $R_{\text {mid }}\left(t_{1}\right)=R_{0}\left(L_{1} / L_{0}\right)^{-3 / 4} \approx 0.58 \mathrm{~mm}$. The corresponding capillary time scale is $t_{\text {cap }}=1.586$ seconds. The axial force in the viscous filament at each instant is computed using eq.(6) and, since the endplates do not move following the initial step displacement that creates the necked configuration, $\dot{L}_{p}(t)=0 \forall t \geq t_{1}$.

In Figure 2(a) we show the evolution in the numerically computed values of the dimensionless force $f(t)$, the dimensionless radius $R_{\text {mid }}(t) / R_{0}$ and the ratio $X(t)$ as the viscous filament necks down and ultimately breaks. Numerical computations are halted at $R_{\text {mid }} / R_{0} \approx 10^{-3}$ due to limits on the numerical resolution possible with $N \sim 1000$ points. It is clear from Figure 2(a) that even though the axial force in the filament and the midpoint radius both decrease to zero in finite time, the ratio given by eq. (8) never approaches the value $X=1$ assumed in eq. (2). In other words, although the filament may be slender, the shape never approaches the axially uniform thread assumed by Entov \& Hinch. Instead the numerically computed solution asymptotically approaches the value $X \approx 0.7127$ characteristic of the similarity solution of Papageorgiou. In his original work, Papageorgiou considered a number of different initial conditions and demonstrated that the dynamical evolution of the bridge was always attracted to this similarity solution. Although we have not performed an extensive 
parametric study, the dimensionless time required for the filament shape to approach this selfsimilar solution will depend on the initial aspect ratio $\Lambda_{0}=L_{0} / R_{0}$ and the magnitude of the stretch $L_{1} / L_{0}$ imposed on the liquid bridge.

In Figure 2(b) we replot the computed data in terms of the dimensional radius and dimensional time. We also indicate on this figure the prediction of the linear theory arising from a local force balance (eq.2) and also the Papageorgiou similarity solution. Figure 2(b) shows that for a viscous Newtonian fluid it is essential to incorporate the axial non-uniformity of the necking fluid filament at all stages of the breakup process. The slenderness of the bridge and the tensile viscous force within the necking column are balanced and both affect the rate of change in the midpoint radius, as described by the similarity solution of Papageorgiou. The simple solution in eq.(2) predicts a critical time to break up of approximately 1.19 seconds whereas the numerical calculation indicates that in fact the break up time should be closer to $3.81 \mathrm{~s}$. This is much closer to the experimental results for the viscous polybutene oil presented by Kolte \& Szabo (see their Figure 7).

Finally, we also show in Figure 2(b) the effects of a non-zero gravitational body force as predicted by the one-dimensional theory (eqs. 5-6). The simulation is performed for a Bond number of $B o=4.6$ to match the finite element computations of Kolte \& Szabo. Gravitational effects significantly affect the evolution of the liquid bridge. At short times there is a global 'slumping' of the column upon the cessation of the imposed stretching that result in a decrease in the midpoint radius (as compared to the $B o=0$ simulation). At intermediate times gravitational forces induce an axial downflow along the fluid column that is fed from the quasi-static fluid reservoir near the upper endplate. This results in a retardation in the rate of necking in $R_{\text {mid }}(t)$. Ultimately, at longer times, the capillary effects in the necked region overcome the gravitational drainage and the filament fails in an identical manner to the $B o=0$ simulation. In this final stage, when the filament is very thin, the rate of decrease in the midpoint radius is again well described by the Papageorgiou similarity solution $(X=0.7127)$. The evolution of the liquid bridge predicted by this one-dimensional Lagrangian simulation is in good agreement with the full timedependent simulation of Kolte \& Szabo although the 'slumping' at short times (when the filament profile is not long and slender) appears to be somewhat over-predicted as a consequence of the inability of the 1D theory to exactly satisfy the no-slip condition at either endplate.

\subsection{Experimental Observation of Breakup in a Microfilament Rheometer}

We have demonstrated that the capillary necking of a viscous Newtonian fluid in a filament rheometer is not well described by eq. (2). The numerical simulations do suggest, however, that the final stages of the breakup of a Newtonian liquid bridge are characterized by 
the simple linear expression in eq. (9) with $X=0.7127$ rather than $X=1$. We now proceed to investigate if this finding is supported by experiment. If observation of the necking process indicates a linear decrease in the radius with time then the use of eq. (9) with $X=0.7127$ should result in a numerical value for the ratio $\sigma / \eta_{s}$ that is consistent with independent measurements of the material properties using a surface tensiometer and a standard torsional rheometer respectively.

We have investigated the capillary breakup of three Newtonian fluids; glycerol (denoted GLY), a silicone oil (PDMS) and a very viscous polybutene oil (PB). Experiments are performed in a new Capillary Breakup Extensional Rheometer (CABER) developed in conjunction with Cambridge Polymer Group (Cambridge, MA). The experimental device utilizes plates of radius $R_{0}=3.0 \mathrm{~mm}$ separated by a distance $L_{0}=2.2 \mathrm{~mm}$ so that the initial aspect ratio is $\Lambda_{0}=0.733$. The plates are separated rapidly using a spring mechanism as in the original design of Bazilevsky et al. (1990) to a final separation of $L_{1}=6.6 \mathrm{~mm}$. The filament midpoint diameter is then monitored using a laser micrometer (Omron, model 24LA-L10) with a calibrated resolution of $20 \mu \mathrm{m} \leq 2 R_{\text {mid }} \leq 5 \mathrm{~mm}$. The micrometer readings are recorded digitally using the LabView data acquisition program along with the temperature in the sample chamber. The global evolution of the column profile is recorded using a CCD camera and macro lens, and sequences of images are digitized using a digital video frame grabbing board.

In Figure 3 we show a sequence of images using glycerol as the test fluid at $T=23.5{ }^{\circ} \mathrm{C}$. The first images show the initial cylindrical liquid bridge configuration and its rapid extensional deformation into a necked configuration. The location of the midpoint radius measurement is indicated by the bright reflection of the laser light-sheet near the axial midplane of the elongated filament. For convenience, and consistency with previous work, time is referenced to the instant when stretching is halted (i.e. we set $t_{1}=0$ ). The subsequent images show the progressive viscocapillary drainage and ultimate breakup of the filament at a critical time $t_{c}=0.349 \pm 0.017$ seconds after the cessation of stretching. The temporal resolution of the singularity is limited by the framing rate of the video camera to 30 frames/second. More accurate identification of the critical time to breakup can be readily ascertained using a high-speed video camera if so desired, but we have not found this to be necessary in identifying the fluid viscosity since the computer acquisition system can obtain data from the laser micrometer at a much faster rate (ca. 1000 samples/sec). The Bond number for this fluid is $B o=1.67$ and the effects of an axial body force can be clearly observed in the breaking of top/bottom symmetry in the bridge resulting in the 'slumping' of fluid discussed in $§ 3.1$.

In Figure 4 we show the midpoint filament diameter $2 R_{\text {mid }}(\mathrm{t})$ for the glycerol sample recorded using the laser micrometer in the CABER. The radius decreases approximately linearly with time in accordance with eq. (9) and hence linear regression can be used to obtain the slope, 
if $X$ is known. Also shown in Figure 4 by the dashed line is the linear solution of eqs (1)-(2) computed using the initial diameter reading $2 R_{1}$ at time $t_{1}$ plus independently measured values of viscosity $\left(\eta_{s}^{(G L Y)}=1.04\right.$ Pa.s $)$ and surface tension $\left(\sigma^{(G L Y)}=0.065 \mathrm{~N} / \mathrm{m}\right)$. It is clear that the slope is significantly different from that observed experimentally. Conversely, if eq. (2) was regressed to the experimental data shown then an erroneous value of the ratio $\sigma / \eta_{s}$ would be obtained. The one-dimensional numerical calculations in $\$ 3.1$ showed that the evolution of a viscous liquid bridge ultimately approaches the similarity solution of Papageorgiou. At earlier times, the slope of the curve (corresponding to the coefficient $(2 X-1) / 6$ in Table 1 and eq. 9) varies continuously with time as indicated in Fig 2. This slow approach to the asymptotic solution has also been shown in the recent full two-dimensional time-dependent calculations of Pozrikidis (1999). It thus remains to determine which part of the experimental curve should be fitted to the similarity solution.

As we have shown in our simulations and the experimental images of Fig. 3 the early stages of the necking are in fact dominated by gravitational drainage which breaks the symmetry demanded by Papageorgiou's solution to the Stokes' equations. Gravitational effects become unimportant when the Bond number becomes much less than unity. If we choose this criterion to correspond to $\rho g\left(2 R_{\text {mid }}(t)\right)^{2} / \sigma \cong 0.1$, then we may expect experimental measurements of viscocapillary thinning in viscous Newtonian bridges to be governed by the similarity solution of Papageorgiou for filament diameters in the range

$$
2 R_{\text {mid }}(t) \leq \sqrt{\frac{\sigma}{10 \rho g}} .
$$

For the case of glycerol, this corresponds to filament diameters less than $0.8 \mathrm{~mm}$. The solid line in Figure 4 is fitted to the corresponding range of data using eq. (9) with $X=0.7127$ taken from Table 1 . The value of the capillary velocity $\left(\sigma / \eta_{s}\right)$ determined by linear regression is then $\sigma / \eta_{s}=66.1 \times 10^{-3} \mathrm{~m} / \mathrm{s}$; which is within $5 \%$ of the ratio computed from the independent measurements of $\sigma$ and $\eta_{s}$.

In Figure 5 we show the results from two additional experiments using silicone oil (PDMS) and polybutene (PB) at $T=23.5^{\circ} \mathrm{C}$. In this plot, the midpoint diameter is shown on a semi-logarithmic scale to better highlight the singular nature of the breakup event and the accuracy of the similarity solution fit to the data in the final stages of the breakup process. The values of the coefficients $\sigma / \eta_{s}$ extracted for each fluid using the Papageorgiou analysis are listed in Table 2 together with the value computed from independent measurements of $\sigma$ and $\eta_{s}$ in our laboratory. An error analysis of at least 5 tests on each fluid indicates that using the analysis outlined above we can achieve a reproducibility of $\pm 10 \%$ ( \pm 2 standard deviation confidence 
interval). Much of this experimental error comes from ensuring a uniform axisymmetric liquid bridge is generated at the start of the test.

\begin{tabular}{|l|c|c|c|cc|}
\hline Fluid & $\sigma[\mathrm{N} / \mathrm{m}]^{(\mathrm{i})}$ & $\eta_{s}[\mathrm{~Pa} . \mathrm{s}]^{(\mathrm{ii})}$ & $\sigma / \eta_{s}[\mathrm{~m} / \mathrm{s}]$ & $\mathrm{CABER} \sigma / \eta_{s}[\mathrm{~m} / \mathrm{s}]\left(T{ }^{\circ} \mathrm{C}\right)$ \\
\hline \hline Glycerin (GLY) & 0.0648 & 1.03 & $62.9 \times 10^{-3}$ & $66.0 \times 10^{-3}$ & $(23.5)$ \\
Silicone oil (PDMS) & 0.0209 & 10.5 & $1.99 \times 10^{-3}$ & $2.02 \times 10^{-3}$ \\
Polybutene H100 (PB) & 0.0304 & 24.0 & $1.26 \times 10^{-3}$ & $1.32 \times 10^{-3}$ \\
\hline
\end{tabular}

Table 2 Values of the material properties for three Newtonian fluids determined from (i) surface tensiometry (using a Krüss K-10 tensiometer); (ii) steady shear rheometry (using a TA Instruments AR1000N controlled stress rheometer). The ratio $\sigma / \eta_{s}$ is compared with the corresponding value obtained from studies of visco-capillary drainage using the modified analysis (eq. 9 with $X=0.7127$ ).

It is clear that for a wide range of viscosities (spanning a number of common viscous liquids) it is possible to use this analysis to accurately extract a numerical value for the ratio of material parameters characterizing the visco-capillary breakup process of a liquid bridge. This value can be combined with one independent measurement of either the surface tension or the viscosity to find a value of the remaining material coefficient.

\section{Conclusions and Discussion}

In the present work we have shown that it is possible to use measurements of the capillary breakup of a viscous Newtonian fluid in order to obtain experimental values for the capillary velocity (i.e. the material property ratio $\sigma / \eta_{s}$ ) that are in quantitative agreement with values determined from rheometry and tensiometry. As the necked region of the liquid bridge evolves under capillary pressure, the shape of the filament becomes progressively closer to the self-similar form first discovered by Papageorgiou (1995). In this regime, the midpoint radius decreases linearly in time according to the evolution equation

$$
R_{\text {mid }}(t)=0.0709 \frac{\sigma}{\eta_{s}}\left(t_{c}-t\right)
$$

and linear regression of eq. (11) to the data can be used to determine $\sigma / \eta_{s}$ and the critical time to breakup, $t_{c}$. 
The simulations of Papageorgiou (1995) and Pozrikidis (1999) demonstrate that the early stages of the breakup process are sensitive to the initial shape of the filament. Our numerical simulations using the one-dimensional Lagrangian formulation of Renardy show that the time required to approach this self-similar regime also depends on other dimensionless parameters such as the Bond number and the aspect ratio of the original sample. However, for sufficiently thin and long threads, the evolution in the necked region will eventually become independent of these external scales and evolve in a self-similar fashion. It would be interesting to perform a numerical study that investigates the inverse question: what is the optimal aspect ratio to use in such a device for typical Bond numbers encountered in experiments $(0.1 \leq B o \leq 10)$ ?

Very close to the breakup event, Brenner et al. (1996) and Eggers (1997) note that inertial effects can no longer be neglected in the fluid, since the local rate of extensional deformation $\left(\dot{\varepsilon} \approx\left(R_{\text {mid }}\right)^{-1} \partial R_{\text {mid }} / \partial t\right)$ diverges. In this region one should thus expect the solution given in eq. (9) to cross over from the inertialess similarity solution with $X=0.7127$, to the universal form discovered by Eggers with $X=0.5912$. Balancing the largest (neglected) inertial terms in the axial momentum equation with the viscous terms retained in the Papageorgiou solution leads to the following estimate for the 'cross-over time' before the singularity:

$$
t^{*}=\frac{\sigma}{\eta_{s} R_{0}}\left(t_{c}-t\right) \sim\left(\frac{\rho \sigma R_{0}}{\eta_{s}^{2}}\right)^{1 /(1-2 \beta)},
$$

where we have taken the initial plate radius $R_{0}$ to be the characteristic external scale entering the viscous solution (Brenner et al. 1996) and the similarity exponent $\beta=0.175$ for the similarity solution of Papageorgiou. The dimensionless quantity on the right-hand-side is the inverse of the Ohnesorge number defined in Table 1. As a result of the high viscosities, the time increments obtained from eq. (12) are very small for the fluids considered in the present work. Using the values given in table 2 we may estimate that for glycerol, inertial effects become important 4.8 milliseconds from the singular break-up event, whilst for PDMS this time is reduced to $14.6 \mu \mathrm{s}$ ! It is thus clear why we do not see this cross-over to the inertial regime in the present experiments. Re-examination of the very careful strobe videography measurements presented by Kowalewski (1986; especially figure 12 therein) using our eq. (9) indicates that the Papageorgiou solution also provides a superior fit to the data than the Eggers solution for most of the observations made.

The recent experimental and numerical studies of capillary breakup phenomena provide confidence in the rheometric capabilities of filament breakup rheometers such as the CABER. It is now possible to interrogate the measured diameter data and systematically extract quantitative 
values for either the characteristic relaxation time constant and the elastic modulus of elastic fluids (Bazilevskii et al. 1997), or the ratio $\sigma / \eta_{s}$ for Newtonian fluids. Of course, such data can be readily extracted from other more-controllable measuring techniques. The principal benefit of capillary breakup measurements in a device such as CABER is that the balance between capillary pressure and viscous or elastic extensional stresses is directly relevant to typical commercial filling and droplet breakup processes involving rheologically-complex materials such as food stuffs (Padmanhaban, 1995), paints, agricultural sprays (Mun \& Boger, 1999) and pressure sensitive adhesives (Tripathi et al. 1999). Of course, for these rheologically-complex fluids, the quantitative interpretation of data is much more difficult; but it is always possible to define an 'apparent extensional viscosity' (Schümmer \& Tebel, 1983). The simplicity of both the experimental test and the subsequent analysis (as outlined in the work of Entov \& Hinch for highly elastic fluids and in the present work for viscous fluids) suggests that such measurements may be of use in quality control applications. In such tests, the principal quantity of interest is the life-time of the fluid filament which sets process parameters such as the batch filling time and, as we show in eq. (10), for Newtonian fluids this is directly connected to the surface tension and viscosity of the filament. For elastic polymer solutions, Bazilevskii et al. (1997) have also used such lifetime measurements to monitor the mechanical degradation of PEO solutions.

As a simple example of the use of the CABER in such applications we show in Figure 6 the evolution in the measured viscosity of glycerol in a sequence of repeated tests in the CABER at $T=24^{\circ} \mathrm{C}$. Reagent grade anhydrous glycerol (100\%) is known to be hygroscopic and following exposure to laboratory air slowly absorbs water vapor. This hygroscopic response is employed commercially in delivery of both foodstuffs (Cisneros-Zevallos et al. 1997) and pharmaceuticals (Hickey \& Mortensen, 1993). As a result of the absorption of water, the viscosity of glycerol progressively decreases with time. In Figure 6(a) repeated tests in the CABER device on the same fluid sample show that as the material ages, it breaks increasingly rapidly. Using eq. (11) the evolution in the capillary velocity $\sigma / \eta_{s}$ with time can be extracted. The variation in surface tension and viscosity of glycerol/water mixtures has been tabulated (Weast, 1984) and as the weight fraction of water increases from $0 \%$ to $12 \%$ the viscosity drops by almost an order of magnitude from $1.03 \mathrm{~Pa}$.s to $0.147 \mathrm{~Pa}$.s. Over the same range of concentrations, the surface tension changes by only 3\%; increasing from 63 to $66 \mathrm{~N} / \mathrm{m}$. Ignoring the weak latter variation, the results can be represented as shown in Figure 6(b). The asymptotic approach to a new constant viscosity value that is $80 \%$ smaller than that obtained for pure glycerol represents approach to a dynamic equilibrium between the glycerol-water fluid mixture and the relative humidity in the laboratory air. 
This simple experiment provides a demonstration of how an experimental device such as the one outlined in the present work can be readily used to monitor temporal changes in the material properties of a fluid which can be difficult to follow in other rheometric devices. The rapid removal/absorption of volatile solvents such as water, methanol etc. from biological fluids and many commercially-important fluid formulations plays an important role in controlling the extensional rheological response and dynamics of processes such as spin-casting, roll-coating and wet-spinning of polymeric fibers. Such an instrument can also be used to monitor the evolution in the extensional characteristics of time-evolving or curing materials such as chemical and physical gels.

\section{Acknowledgments}

The authors would like to thank the DuPont Educational Aid program for financial support of this research, and Cambridge Polymer Group for assistance in developing the experimental hardware and software used in this work. The authors would like to acknowledge numerous illuminating conversations with Jens Eggers and Michael Brenner and the insightful comments of the anonymous referees. 


\section{References}

Anna, S.L., McKinley, G.H. Quantifying the Stretching and Breakup of Dilute polymer Solutions in Two Different Filament Stretching Experiments, paper 175a, A.I.Ch.E. Annual Meeting, Dallas, Nov. 1999.

Bazilevsky, A.V., Entov, V.M. and Rozhkov, A.N., "Liquid Filament Microrheometer and Some of its Applications", Third European Rheology Conference, D. R. Oliver (ed.), Elsevier Applied Science, 1990.

Bazilevskii, A.V., Entov, V.M., Lerner, M.M. and Rozhkov, A.N., Failure of Polymer Solution Filaments, Polymer Science Ser. A (translated from Vysokomolekulyarnye Soedineniya Ser. A pp. 474-482), 39(3), (1997), 316-324.

Berg, S., Kröger, R. and Rath, H.J., Measurement of Extensional Viscosity by Stretching Large Liquid Bridges in Microgravity, J. Non-Newtonian Fluid Mech., 55, (1994), 307-319.

Bogy, D.B., Drop Formation in a Circular Liquid Jet, Ann. Rev. Fluid Mech., 11, (1979), 207228.

Bousfield, D.W., Keunings, R., Marrucci, G. and Denn, M.M., Nonlinear Analysis of the Surface-Tension Driven Breakup of Viscoelastic Fluid Filaments, J. Non-Newt. Fluid Mech., 21, (1986), 79-97.

Brenner, M.P., Lister, J.R. and Stone, H.A., Pinching Threads, Singularities and the Number 0.0304..., Phys. Fluids, 8(11), (1996), 2827-2836.

Cisneros-Zevallos, L., Saltveit, M.E. and Krochta, J.M., Hygroscopic Coatings Control Surface White Discoloration of Peeled (Minimally Processed) Carrots During Storage, J. Food Science, 62(2), (1999), 363.

Entov, V.M. and Hinch, E.J., Effect of a Spectrum of Relaxation Times on the Capillary Thinning of a Filament of Elastic Liquid, J. Non-Newt. Fluid Mech., 72(1), (1997), 31-54.

Entov, V.M. and Yarin, A.L., Influence of Elastic Stresses in the Capillary Breakup of Dilute Polymer Solutions, Fluid Dyn. (translated from Izvestiya Akademii Nauk SSSR, Mekhanika Zhidkosti i Gaza), 19(1), (1984), 21-29.

Eggers, J., Nonlinear Dynamics and Breakup of Free-Surface Flows, Rev. Mod. Phys., 69(3), (1997), 865-929.

Gaudet, S., McKinley, G.H. and Stone, H.A., Extensional Deformation of Newtonian Liquid Bridges, Phys. Fluids, 8(10), (1996), 2568-2579.

Gillette, R.D. and Dyson, D.C., Stability of Fluid Interfaces of Revolution Between Equal Solid Circular Plates, Chem. Eng. J., 2, (1971), 44-54. 
Goldin, M., Yerushalmi, H., Pfeffer, R. and Shinnar, R., Breakup of a Laminar Capillary Jet of a Viscoelastic Fluid, J. Fluid Mech., 38(4), (1969), 689-711.

Harlen, O.G., Presentation at the Isaac Newton Institute Program on The Dynamics of Complex Fluids, Apr. 1996.

Hickey, A.J. and Mortensen, T.B., Behavior of Hygroscopic Pharmaceutical Aerosols and the Influence of Hydrophobic Additives, Pharmaceutical Research, 10(1), (1993), 1-7.

Kolte, M.I. and Szabo, P., Capillary Thinning of Polymeric Filaments, J. Rheology, 43(3), (1999), 609-626.

Kowalewski, T.A., On the Separation of Droplets from a Liquid Jet, Fluid Dyn. Res., 17, (1996), 121-145.

Kröger, R., Berg, S., Delgado, A. and Rath, H.J., Stretching Behavior of Large Polymeric and Newtonian Liquid Bridges in Plateau Simulation, J. Non- Newt. Fluid Mech., 45, (1992), 385400.

Liang, R.F. and Mackley, M.R., Rheological Characterization of the Time and Strain Dependence for Polyisobutylene Solutions, J. Non- Newt Fluid Mech., 52 (1994), 387-405.

Matta, J.E. and Tytus, R.P., Liquid Stretching Using a Falling Cylinder, J. Non-Newt. Fluid Mech., 35, (1990), 215-229.

Mun, R.P., Young, B.W. and Boger, D.V., Atomization of Dilute Polymer Solutions in Agricultural Spray Nozzles, J. Non-Newt. Fluid Mech., 83(1-2), (1999), 163-178.

Padmanabhan, M., Measurement of Extensional Viscosity of Viscoelastic Liquid Foods, J. Food Eng., 25, (1995), 311-327.

Papageorgiou, D.T., On the Breakup of Viscous Liquid Threads, Phys. Fluids, 7(7), (1995), 1529-1544.

Plateau, J.A.F., "Experimental and Theoretical Researches on the Figures of Equilibrium of a Liquid Mass Withdrawn from the Action of Gravity", Ann Rep. Smithsonian Institution, 1863.

Pozrikidis, C., Capillary Instability and Breakup of a Viscous Thread, J. Eng. Math., 36, (1999), 255-275.

Renardy, M., Some Comments on the Surface-Tension Driven Breakup (or the lack of it) of Viscoelastic Jets, J. Non-Newt. Fluid Mech., 51, (1994), 97-107.

Renardy, M., A Numerical Study of the Asymptotic Evolution and Breakup of Newtonian and Viscoelastic Jets, J. Non-Newt. Fluid Mech., 59, (1995), 267-282. 
Schümmer, P. and Tebel. K.H., Instability of Jets of Non-Newtonian Fluids, in Rheology Vol. 2: Fluids, pp. 87-92. Plenum Press (New York) 1980.

Schümmer, P. and Tebel. K.H., A New Elongational Rheometer for Polymer Solutions, J. NonNewt. Fluid Mech., 12, (1983), 331-347.

Shipman, R.W.G., Denn, M.M. and Keunings, R., Mechanics of the 'Falling Plate' Extensional Rheometer, J. Non-Newtonian Fluid Mech., 40, (1991), 281-288.

Spiegelberg, S.H., Ables, D.C. and McKinley, G.H., The Role of End-Effects on Measurements of Extensional Viscosity in Viscoelastic Polymer Solutions With a Filament Stretching Rheometer, J. Non-Newt. Fluid Mech., 64(2-3), (1996), 229-267.

Slobozhanin, L.A. and Perales, J.M., Stability of Liquid Bridges between Equal Disks in an Axial Gravity Field, Phys. Fluids A, 5(6), (1993), 1305-1314.

Szabo, P., Transient Filament Stretching Rheometer I: Force Balance Analysis, Rheol. Acta, 36(3), (1997), 277-284.

Tirtaatmadja, V. and Sridhar, T., A Filament Stretching Device for Measurement of Extensional Viscosity, J. Rheol., 37(6), (1993), 1081-1102.

Tjahjadi, M., Ottino, J.M. and Stone, H.A., Estimating Interfacial Tension via Relaxation of Drop Shapes and Filament Breakup, A.I.Ch.E. J., 40(3), (1994), 385-394.

Tripathi, A., Whittingstall, P. and McKinley, G.H., Using Filament Stretching Rheometry to Predict Strand Formation and "Processability" in Adhesives and Other Non-Newtonian Fluids, Rheol. Acta, (2000), in press.

Yarin, A.L., Free Liquid Jets and Films: Hydrodynamics \& Rheology, (Interaction of Mechanics and Mathematics Series), Longman-Wiley, New York, 1993.

Weast, R. C. (ed.), Handbook of Chemistry and Physics, $64^{\text {th }}$ Edition, 1984, CRC Press.

Yao, M., McKinley, G.H. and Debbaut, B., Extensional Deformation, Stress Relaxation and Necking Failure of Viscoelastic Filaments, J. Non-Newt. Fluid Mech., 79(3) (1998), 469-501.

Yao, M., Spiegelberg, S.H. and McKinley, G.H., Fluid Dynamics of Weakly Strain-Hardening Fluids in Filament Stretching Devices, J. Non-Newt. Fluid Mech., 89(1-2), (2000), 1-43. 


\section{List of Figures}

Figure 1 Schematic diagram of operation of a liquid filament rheometer and different conceptual models for the subsequent visco-capillary drainage of a fluid column: $(a)$ initial configuration of liquid bridge, $t<t_{0} ;(b)$ imposed stretching deformation, $t_{0} \leq t \leq t_{1} ;(c)$ cessation of stretching and measurement of visco-elasto-capillary drainage of the slender necked filament using a laser micrometer at the axial midplane $t \geq t_{1} ;(d)$ axially uniform fluid thread model described by equations (1) \& (2).

Figure 2. Numerical simulations of visco-capillary drainage in a filament rheometer for a fluid with $t_{c a p}=1.586 \mathrm{~s}$, and $R_{1}=1.25 \mathrm{~mm}$. (a) Midpoint radius $R_{\text {mid }}(t) / R_{0}$, tensile force $f(t)=F_{z}(t) /\left(2 \pi \sigma R_{1}\right)$ and the ratio $X(t) ;(b)$ The effect of gravity $B o=0(\square) ; B o=4.6(\bigcirc)$ and the predictions of the similarity solutions of Entov \& Hinch and Papageorgiou.

Figure 3 Sequence of images showing the initial configuration and extensional deformation of a viscous glycerol filament followed by the onset of visco-capillary drainage and ultimately breakup at a critical time $t_{c}=0.349 \pm 0.017 \mathrm{~s}$. The diameter of the endplates is $2 R_{\text {mid }}=5.92 \mathrm{~mm}$ and the Bond number for Glycerol is $B o=1.67$.

Figure 4 The midpoint diameter $2 R_{\text {mid }}(t)$ measured with a laser micrometer for glycerol together with the predictions of the viscous thread theory ( $X=1$, shown by the broken line) and with the asymptotic prediction of the similarity solution $(X=0.7127)$ of Papageorgiou $(-\longrightarrow)$.

Figure 5 The experimentally-measured midpoint diameter $2 R_{\text {mid }}(t)$ shown on a semilogarithmic scale for two additional viscous Newtonian fluids: $(\bigcirc)$ polybutene (PB) and $(\square)$ silicone oil (PDMS) together with the appropriate fits to equation (11).

Figure 6 Absorption of water vapor across the free surface of a liquid bridge of anhydrous glycerol results in an increase in the capillary velocity and a decrease in the time to breakup as shown in (a). The viscosity of the fluid sample approaches a constant value after approximately 5 minutes exposure to humid air. 


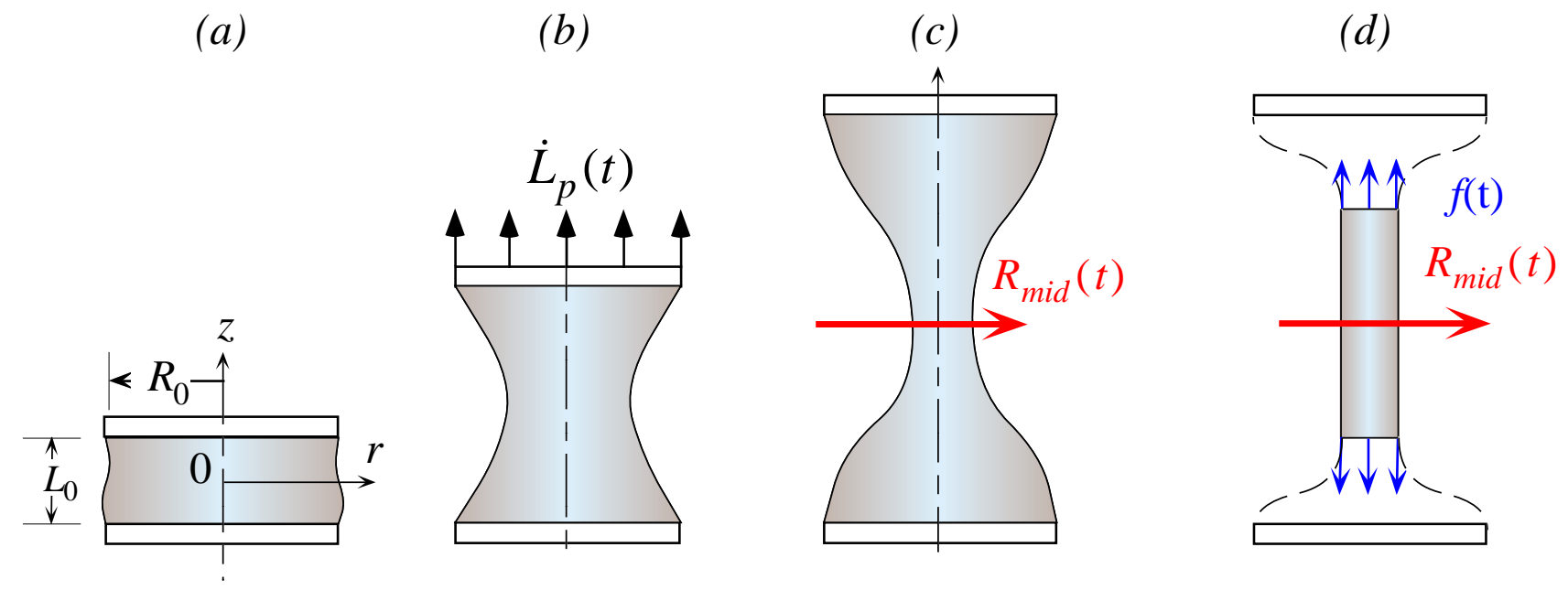

Figure 1 

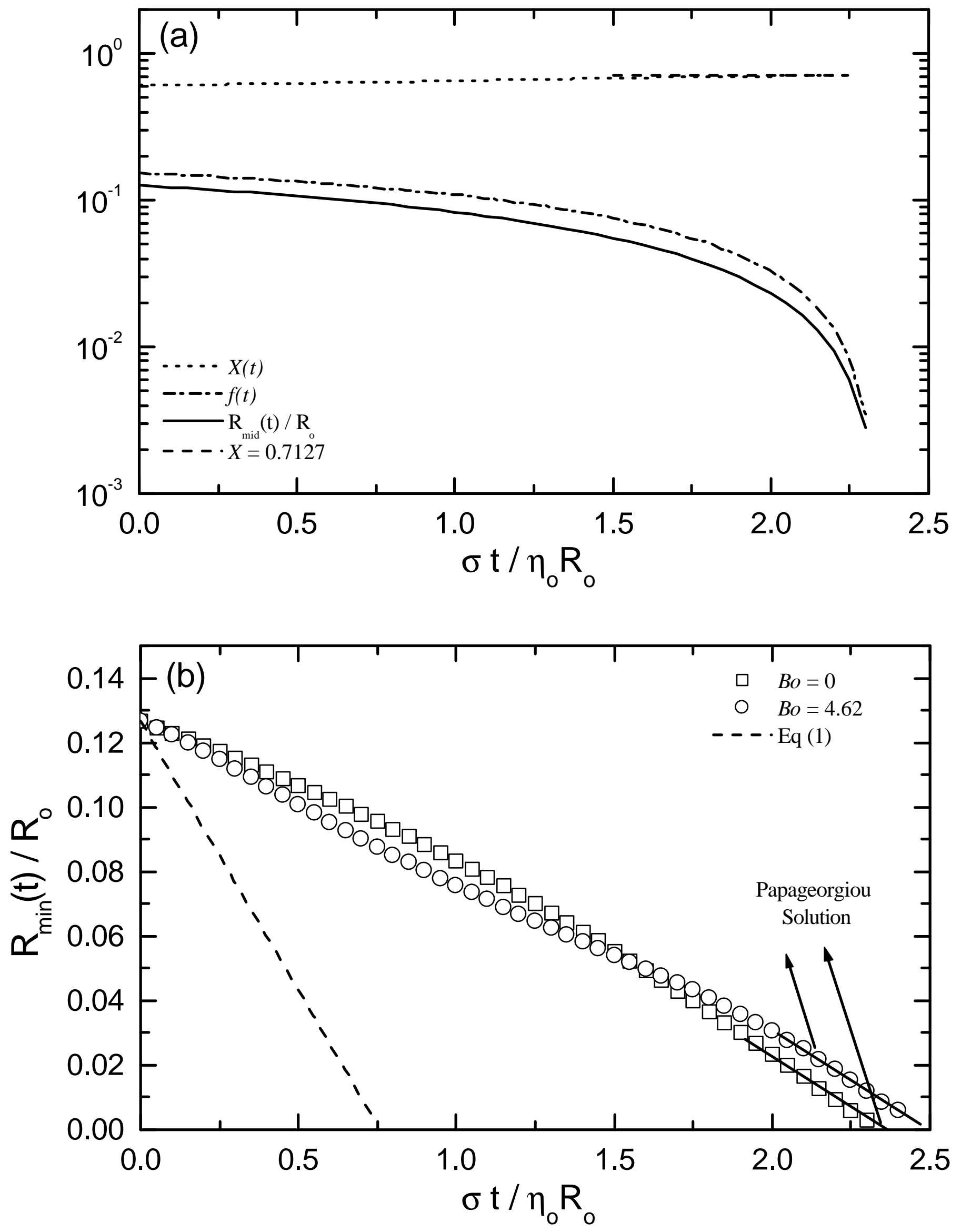


\section{Glycerol Filament Breakup}
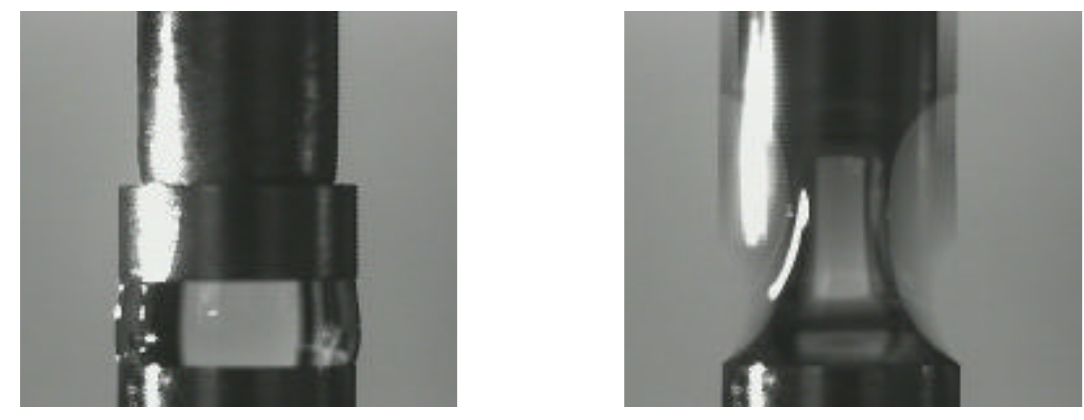

$0 \mathrm{~s}$

$0.100 \mathrm{~s}$

$0.166 \mathrm{~s}$
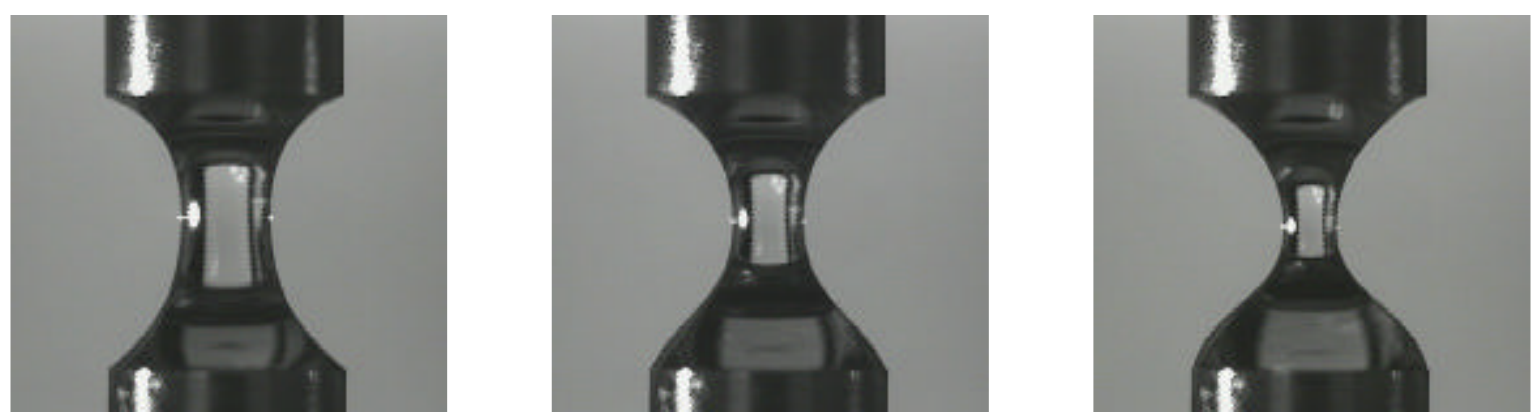

$0.233 \mathrm{~s}$

$0.300 \mathrm{~s}$

$0.333 \mathrm{~s}$
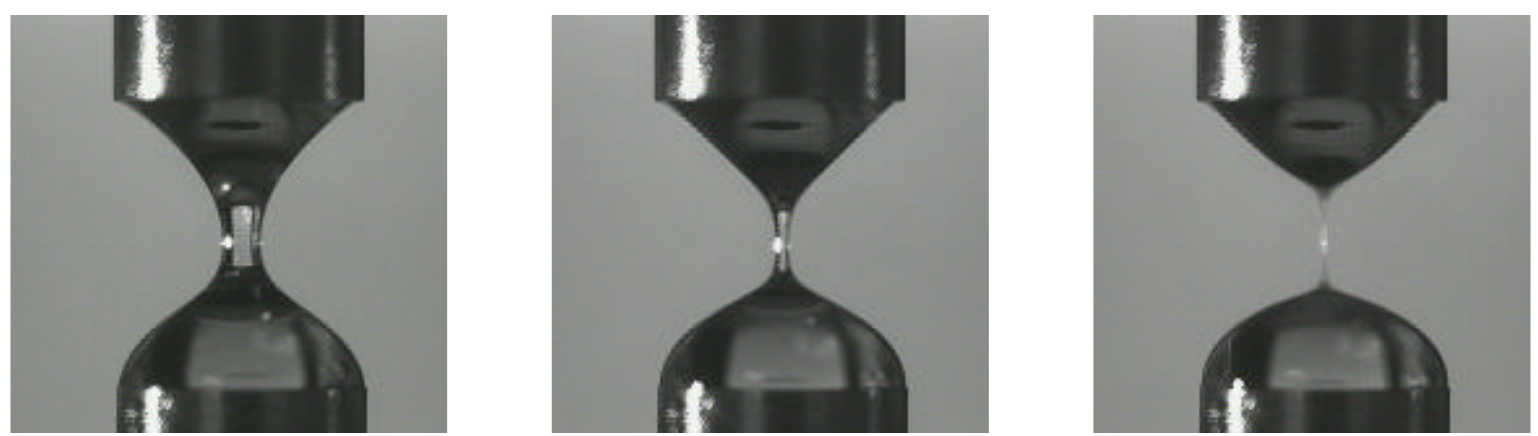


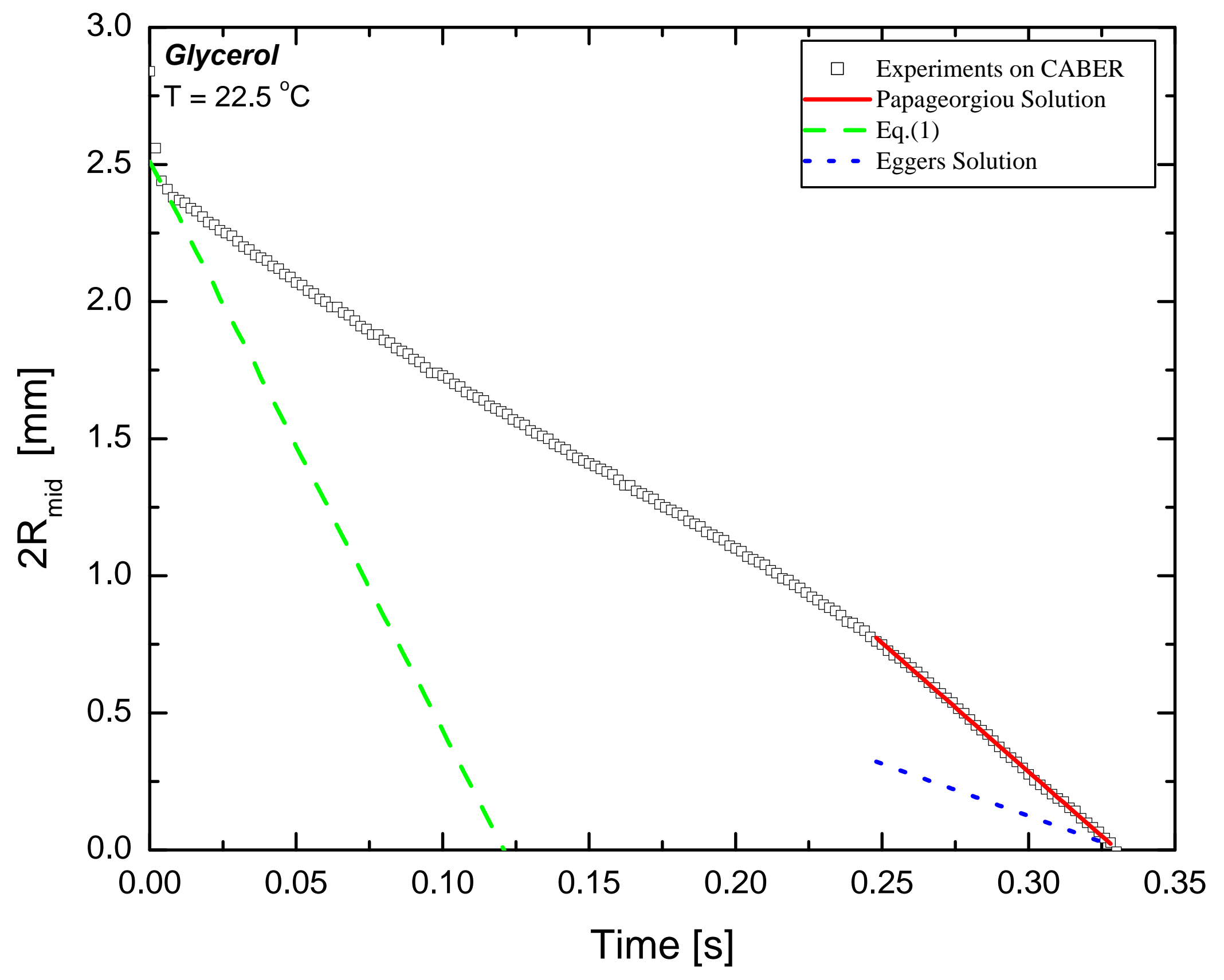




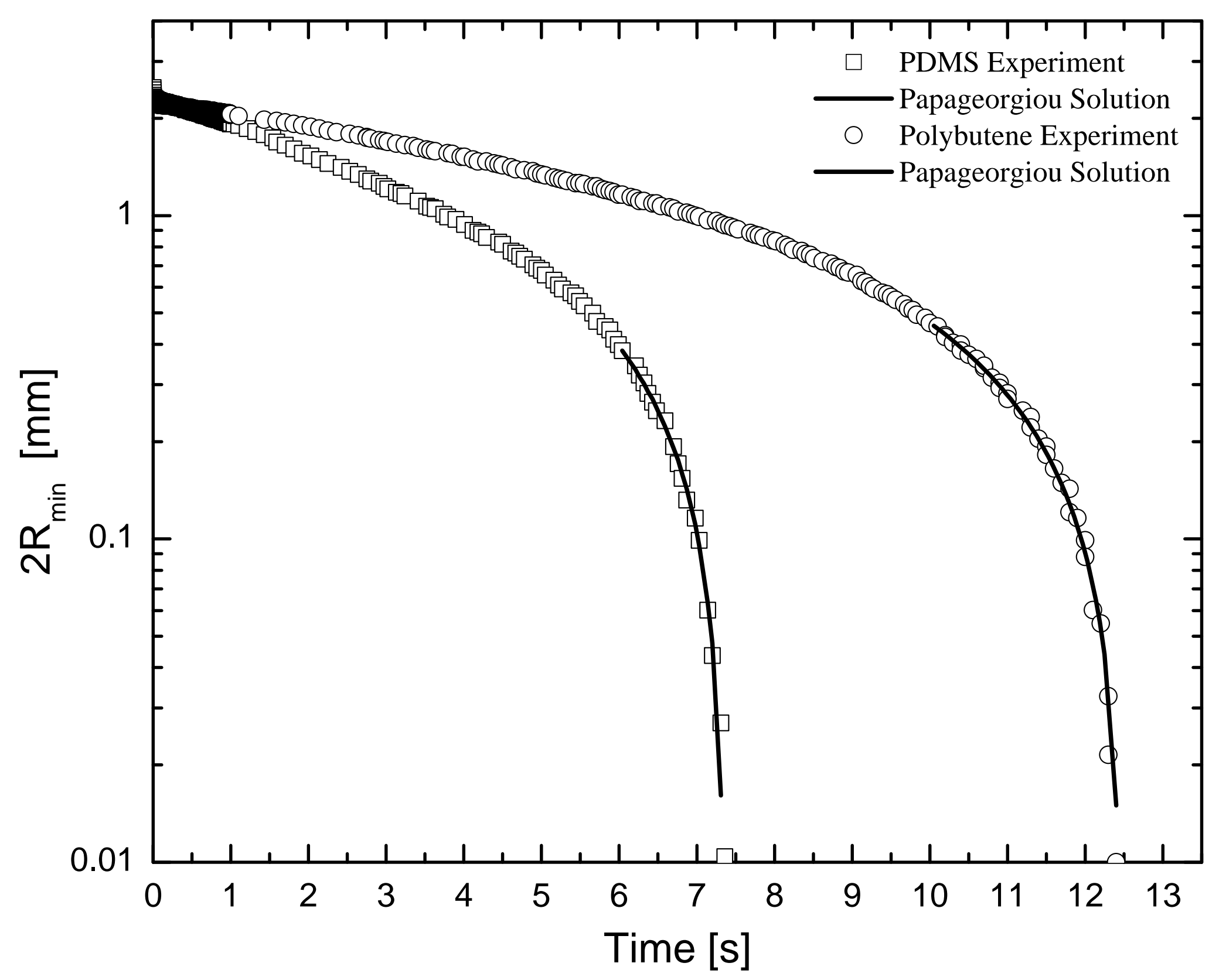



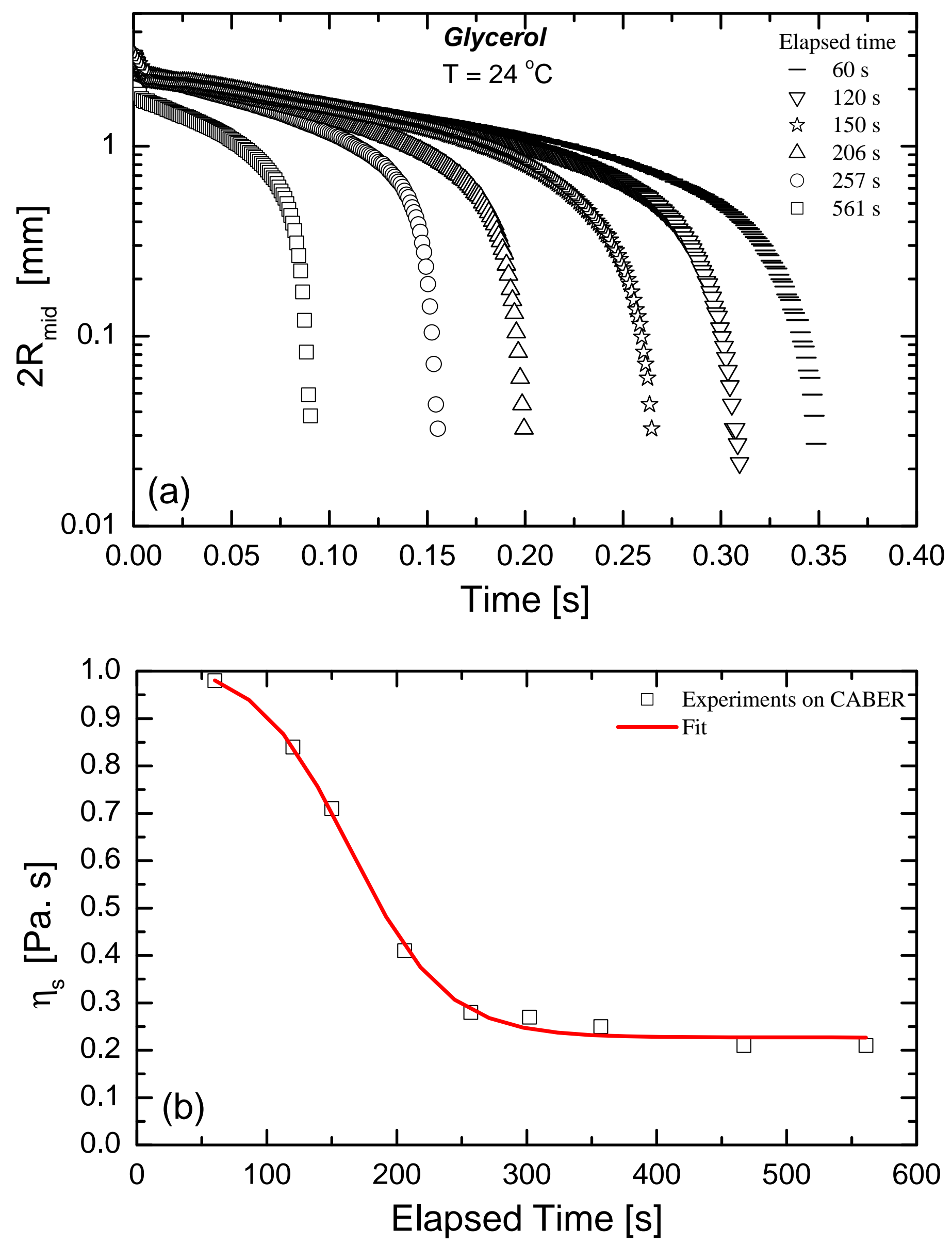